ESAIM: PROCEEDINGS, December 2013, Vol. 42, p. 10-19

A. Heibig, F. Filbet, L. I. Palade, Editors

\title{
SURFACE OPTIMIZATION AND NEW CAVITATION MODEL FOR LUBRICATED FLOW
}

\author{
ERIC DALISSIER ${ }^{1}$
}

\begin{abstract}
Le système piston/chemise/segment est le siège d'une partie importante des pertes en frottement du moteur (de l'ordre de $7 \%$ de l'énergie fournie par le moteur [1]). Une des pistes étudiées pour diminuer ces frottements consiste à introduire des rugosités à la surface de la chemise. Ces rugosités servent localement de réservoir au lubrifiant et permettent de limiter les contacts entre les segments et la chemise et donc de diminuer le frottement. Un des buts de notre travail était d'optimiser ces rugosités de surface en modélisant le système segment/chemise en présence de lubrifiant.
\end{abstract}

\section{INTRODUCTION}

Sous un certain nombre d'hypothèses géométriques, en négligeant les effets thermiques, la possibilité pour les surfaces de présenter des cas de grippage (en imposant une hauteur d'huile suffisante) et en adoptant la méthodologie de Kumar et Brooker [5], le problème se ramène à calculer l'évolution de la hauteur du segment $X(t)$ par rapport à la surface de la chemise (supposée fixe) qui vérifie :

$$
\vec{F}_{\text {imposée }}+\vec{F}_{\text {hydrodynamique }}+\vec{F}_{\text {contact }}=M \frac{\mathrm{d}^{2} \vec{X}}{\mathrm{~d} t^{2}}
$$

où $M$ est la masse du segment.

L'équation (1) comporte en plus des forces imposées deux termes qui devront être calculés à chaque étape de temps :

- une force de frottement hydrodynamique due au frottement du lubrifiant qui se trouve entre le segment et la chemise :

${ }^{1}$ 51, rue des mathématiques, DOMAINE UNIVERSITAIRE, BP 53, 38041 Grenoble cedex 9, email : edalissier@gmail.com

(c) EDP Sciences, SMAI 2013 


$$
\begin{gathered}
\vec{F}_{\text {frottement hydro }}=\left(\begin{array}{l}
\tau_{x y} \\
\tau_{y z} \\
0
\end{array}\right)=\left(\begin{array}{l}
\mu \frac{\partial u_{x}}{\partial z} \\
\mu \frac{\partial u_{y}}{\partial z} \\
0
\end{array}\right) \\
F_{\text {frottement hydro }}=\iint_{\Omega} \tau_{x y}=\iint_{\Omega} \mu\left(\frac{1}{2 \mu} h \frac{\partial p}{\partial x}-\frac{u \theta}{h}\right) d x d y
\end{gathered}
$$

Ce frottement depend de la pression et de la saturation $\theta$ qui seront calculées à l'aide de l'équation de Reynolds [2] - [3] et dont la résolution dépend de $X$.

- une force de contact qui devient importante lorsque le segment et la chemise sont suffisamment proches et que des contacts locaux élastiques ont lieu au niveau des rugosités. Cette force de contact sera prise en compte par le modèle de Greenwood-Tripp [4] qui suppose une répartition gaussienne des aspérités.

\section{Modèle de lubrificAtion pour le Système Piston/SEgment/Chemise.}

\section{1. Équations pour la résolution du problème hydrodynamique (modèle JFO).}

Ce modèle correspond à un problème à frontière libre pour tenir compte de l'aspect diphasique du fluide lié à la présence de cavitation (lorsque la pression interne devient inférieure à la pression de vapeur saturante). Le domaine de calcul est divisé en $2, \Omega_{+}$où $p>0$ et $\theta=1$ et $\Omega_{0}$ où $p=0$ et $\theta<1$.

Pour une vitesse relative $u$ des deux surfaces séparées par une distance $h(x, t)$ qui inclut $X(t)$ et les rugosités, la pression $p(x, t)$ et la saturation $\theta(x, t)$ verifient (cf. Figure 1a) :

$$
\begin{array}{rr}
\frac{\partial}{\partial t}(h \theta)+\frac{\partial}{\partial x}\left(\frac{u}{2} h \theta\right)=\operatorname{div}\left(\frac{h^{3}}{12 \mu} \nabla p\right) & \text { sur } \Omega=\Omega_{+} \cup \Omega_{0} \\
\frac{h^{3}}{12 \mu} \frac{\partial p}{\partial n}=\left(1-\theta^{-}\right) h\left[u_{n}-q_{n}\right] & \text { sur la frontière } \Sigma=\Omega_{+} \cap \Omega_{0}
\end{array}
$$

où $\mu$ est la viscosité, $n$ la normale à l'interface, $u_{n}$ et $q_{n}$ les projections sur la normale de la vitesse du fluide et de la vitesse de la frontière. Un exemple de solution est donnée sur la Figure 1b. 

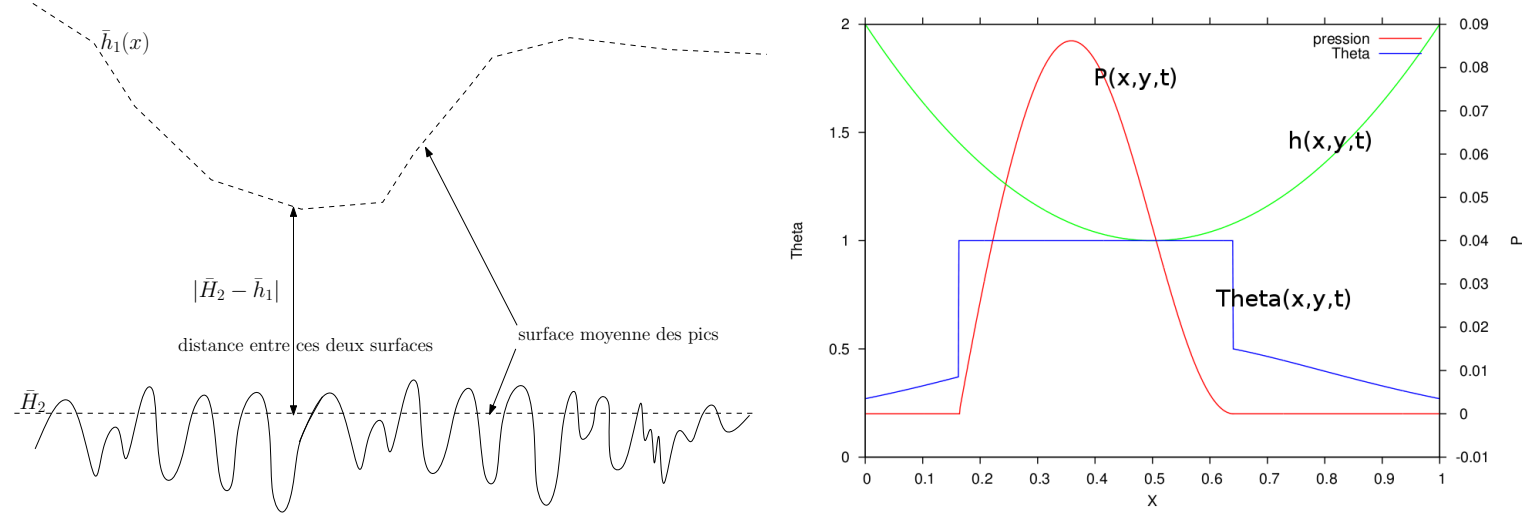

(a) Représentation de la distance entre les 2 surfaces (b) Représentation des différentes variables du modèle JFO moyennes

Elrod-Adams

FiguRE 1. Représentations des différentes variables intervenant dans les deux modèles utilisés

\subsection{Equations pour la force de contact}

Le calcul de la force de contact se fait en utilisant le modèle statistique de Greenwood-Tripp [4] qui suppose une répartition gaussienne des aspérités (figure 1(a)) :

$$
\begin{array}{r}
F_{\text {contact }}(x)=K E^{\prime} \frac{1}{\sqrt{2 \pi}} \int_{\lambda}^{\infty}(u-\lambda(x))^{\frac{5}{2}} e^{-\frac{u^{2}}{2}} d u \\
W_{\text {contact }}=\int_{\mathscr{D}} F_{\text {contact }}(x) d x
\end{array}
$$

avec :

$$
\begin{aligned}
H_{2} & =\sqrt{\int h_{2}(x)^{2} d x} & \sigma=\sqrt{\int\left(h_{2}(x)-H_{2}\right)^{2} d x} & \lambda(x)=\frac{H_{2}-h_{1}(x)}{\sigma} . \\
K T & =\frac{8 \pi}{5}(\eta \beta \sigma) K_{\mathrm{GW}} & \frac{1}{E^{\prime}}=\frac{1-\nu_{1}^{2}}{E_{1}}+\frac{1-\nu_{2}^{2}}{E_{2}} & K_{\mathrm{GW}}=\frac{2 \sqrt{2}}{3}(\eta \beta \sigma) \sqrt{\frac{\sigma}{\beta}}
\end{aligned}
$$

La force de contact est pratiquement nulle lorsque la distance $\lambda(x)>4$, aussi nous ne calculons cette force de contact que pour les points vérifiant :

$$
\left\{(x, y) \in \Omega \mid H_{2}-h_{1}(x, y)<4 \sigma\right\} \quad \text { "zone de contact" }
$$

où $\sigma$ est l'écart type des hauteurs des rugosités de la surface, calculé à partir d'un plan moyen.

\subsection{Prise en compte des rugosités expérimentales}

Des échantillons de surface ont été obtenus par Renault S.A. Ils permettent d'obtenir une description précise d'une surface de $2 \times 2 \mathrm{~mm}$ avec un pas de $2 \mu \mathrm{m}$. Cependant une telle surface n'est pas suffisante pour décrire ce qui se passe entre le segment et la chemise. Il nous faut donc à partir de ce petit échantillon construire une 
surface plus importante. Pour cela, nous avons proposé plusieurs procédures basées sur l'extraction d'un "motif" géomètrique, différent pour chaque échantillon, et qui permet une duplication de la surface (cf. Figure 2).

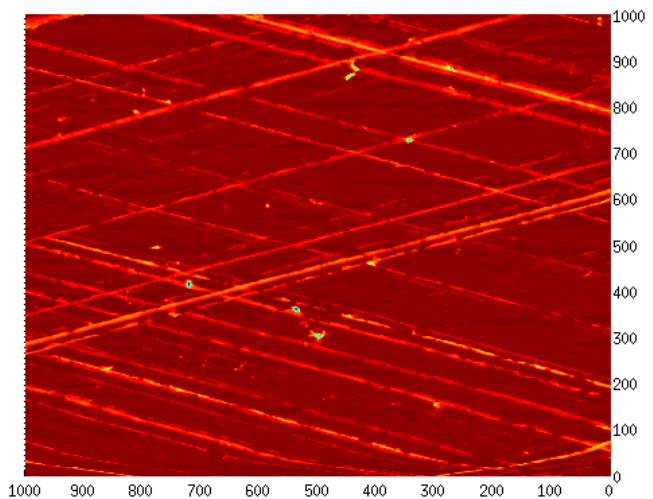

(a) Surface L3 originale

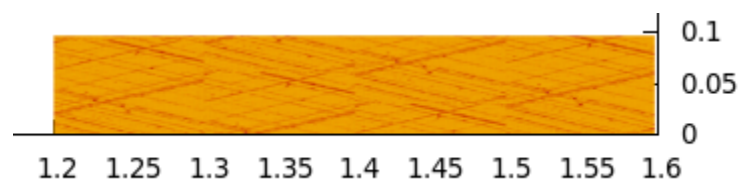

(c) Surface L3 translaté

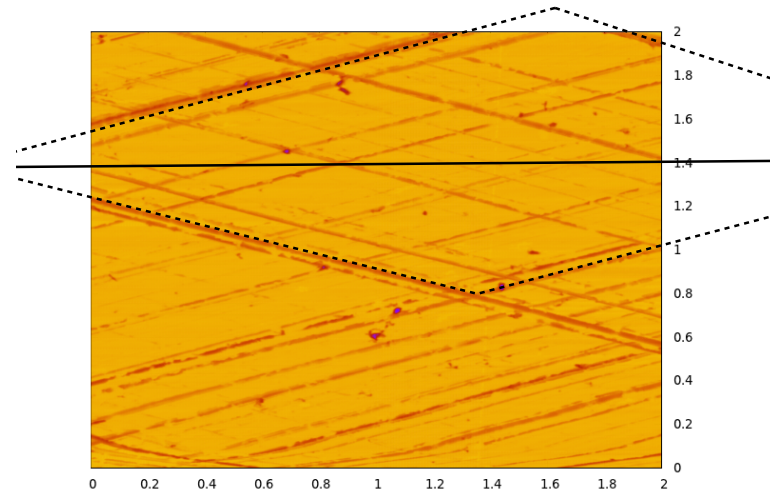

(b) Motif de la surface L3 originale

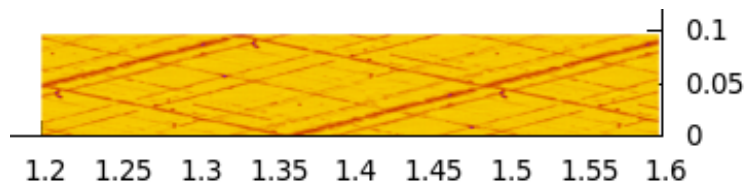

(d) Surface L3 copie par motifs

Figure 2. Différentes méthodes de duplication de surfaces

\subsection{Méthode de résolution pour le problème hydrodynamique}

Nous détaillons ici la mèthode de résolution proposée dans [7] pour la résolution du modèle JFO. 
Trouver $P, \theta$ tel que :

$$
\begin{array}{r}
\Omega_{+}=\{(x, y) \mid p(x, y, t)>0\} \\
\Omega_{0}=\{(x, y) \mid p(x, y, t)=0\} \\
\frac{\partial}{\partial t}(h \theta)+\frac{\partial}{\partial x}\left(\frac{u}{2} h \theta\right)=\operatorname{div}\left(h^{3} \nabla p\right) \\
\theta=1 \text { et } p>0 \\
\theta \in] 0,1[\text { et } \bar{p}=0 \\
p(0, y, t)=0 \\
p(L, y, t)=p_{a} \\
p(x, 0, t)=p(x, B, t) \\
\frac{h^{3}}{2} \frac{\partial p}{\partial n}=\left(1-\theta^{-}\right) h\left[u_{n}-q_{n}\right]
\end{array}
$$

Pour la discrétisation du problème, une discrétisation classique d'ordre 2 en différences finies est utilisées pour les dérivées en pression:

$$
\begin{aligned}
\frac{\partial}{\partial x}\left(h^{3} \frac{\partial p}{\partial x}\right)\left(x_{i}, y_{j}, t^{n}\right)=\frac{1}{2 \Delta} & \left(P_{i+1, j}^{n}\left(h_{i+1, j}^{n 3}+h_{i, j}^{n 3}\right)\right. \\
+ & P_{i, j}^{n}\left(-h_{i-1, j}^{n 3}-2 h_{i, j}^{n 3}-h_{i+1, j}^{n 3}\right) \\
+ & \left.P_{i-1, j}^{n}\left(h_{i+1, j}^{n 3}+h_{i, j}^{n 3}\right)\right)
\end{aligned}
$$

La méthode d'Upwind est utilisée pour la dérivée d'ordre 1 en espace :

$$
\frac{\partial}{\partial x}(\theta h)\left(x_{i}, y_{j}, t^{n}\right)=\frac{h_{i, j}^{n} \theta_{i, j}^{n}-h_{i-1, j}^{n} \theta_{i-1, j}^{n}}{\Delta x}
$$

Enfin pour la discrétisation en temps, nous avons utilisé un schéma implicite donc, nous avons :

$$
\frac{\partial \theta h}{\partial t}=\frac{\theta_{i, j}^{n} h_{i, j}^{n}-\theta_{i, j}^{n-1} h_{i, j}^{n-1}}{\Delta t}
$$

A l'aide de conditions sur la pression et la saturation dans chaque zone, nous allons résoudre le problème :

$$
\begin{array}{cl}
\text { Dans } \Omega_{0} \text { (zone cavité) } & P_{i, j}^{n}=0 \text { et } 0 \leq \theta_{i, j}^{n}<1 \\
\text { Dans } \Omega_{+} \text {(zone active) } & P_{i, j}^{n}>0 \text { et } \theta_{i, j}^{n}=1
\end{array}
$$


Pour cela une méthode de point fixe de type Gauss-Seidel a été utilisée permettant de corriger $P$ et $\theta$ afin qu'ils restent dans leur domaine de définition. Nous avons :

Sur la zone $\Omega_{+, n-1}$ où $P^{n-1,0}>0$ et $\theta^{n-1,0}=1$, on résout l'expression de la pression pour $\theta$ fixé :

$$
\begin{aligned}
P_{i, j}^{n, k+1}=-\frac{1}{\left(B_{2}+B_{5}\right)} & \left(B_{1} P_{i-1, j}^{n, k}+B_{3} P_{i+1, j}^{n, k}+B_{4} P_{i, j-1}^{n, k}+B_{6} P_{i, j+1}^{n, k}\right. \\
& \left.+\left(A_{1}+A_{3}\right) \theta_{i, j}^{n, k}+A_{2} \theta_{i, j}^{n-1, k}+A_{4} \theta_{i-1, j}^{n, k}\right)
\end{aligned}
$$

Sur la zone $\Omega_{0, n-1}$ où $P^{n-1,0}=0$ et $\theta^{n-1,0}<1$, on résout l'expression en $\theta$ à pression fixée :

$$
\begin{aligned}
\theta_{i, j}^{n, k+1}=-\frac{1}{\left(A_{1}+A_{3}\right)} & \left(B_{1} P_{i-1, j}^{n, k}+\left(B_{2}+B_{5}\right) P_{i, j}^{n, k}+B_{3} P_{i+1, j}^{n, k}+B_{4} P_{i, j-1}^{n, k}+B_{6} P_{i, j+1}^{n, k}\right. \\
& \left.+A_{2} \theta_{i, j}^{n-1, k}+A_{4} \theta_{i-1, j}^{n, k}\right)
\end{aligned}
$$

où, $k$ : le nombre d'approximation de $P^{n}$

avec :

$$
\begin{aligned}
B_{1} & =\frac{1}{\Delta x^{2}}\left(h_{i, j}^{n 3}+h_{i-1, j}^{n 3}\right) \\
B_{2} & =\frac{1}{\Delta x^{2}}\left(-h_{i-1, j}^{n 3}-2 h_{i, j}^{n 3}-h_{i+1, j}^{n 3}\right) \\
B_{3} & =\frac{1}{\Delta x^{2}}\left(h_{i, j}^{n 3}+h_{i+1, j}^{n 3}\right) \\
B_{4} & =\frac{1}{\Delta y^{2}}\left(h_{i, j}^{n 3}+h_{i, j-1}^{n 3}\right) \\
B_{5} & =\frac{1}{\Delta y^{2}}\left(-h_{i, j+1}^{n 3}-2 h_{i, j}^{n 3}-h_{i, j-1}^{n 3}\right) \\
B_{6} & =\frac{1}{\Delta y^{2}}\left(h_{i, j}^{n 3}+h_{i, j+1}^{n 3}\right)
\end{aligned}
$$

$$
\begin{aligned}
& A_{1}=\frac{-h_{i, j}^{n 3}}{\Delta t} \\
& A_{2}=\frac{-h_{i, j}^{n-13}}{\Delta t} \\
& A_{3}=\frac{-u \cdot h_{i, j}^{n 3}}{\Delta x_{3}} \\
& A_{4}=\frac{u \cdot h_{i-1, j}^{n}}{\Delta x}
\end{aligned}
$$

$\left(\theta_{i, j}^{n}, P_{i, j}^{n}\right)$ sont calculés à partir d'une suite d'approximation $\left(\theta_{i, j}^{n, k}, P_{i, j}^{n, k}\right)$ tel que :

$$
\left(\theta_{i, j}^{n}, P_{i, j}^{n}\right)=\lim _{k \rightarrow+\infty}\left(\theta_{i, j}^{n, k}, P_{i, j}^{n, k}\right)
$$

et on initialise le système avec : 


$$
\begin{gathered}
\theta_{i, j}^{n, 0}=\theta_{i, j}^{n-1} \\
P_{i, j}^{n, 0}=P_{i, j}^{n-1}
\end{gathered}
$$

où $\left(P_{i, j}^{n-1}, \theta_{i, j}^{n-1}\right)$ sont connues.

A chaque approximation de $\left(\theta_{i, j}^{n, k+1}, P_{i, j}^{n, k+1}\right)$, on vérifie que $P_{i, j}^{n, k+1} \geq 0$ et $\theta_{i, j}^{n, k+1} \in[0,1]$ si ce n'est pas le cas alors on corrige de la manière suivante :

- si $P_{i, j}^{n, k+1}>0$ alors $\theta_{i, j}=1$ sinon $P_{i, j}=0$.

- si $\theta_{i, j}^{n, k+1}<1$ alors $P_{i, j}=0$ sinon $\theta_{i, j}=1$.

Pour la résolution totale du problème, à chaque pas de temps, la position du segment par rapport à la chemise est connue, on peut donc résoudre l'équation pour calculer les forces hydrodynamiques, puis on calcule la force de contact. Cela nous donnera une force verticale qui nous permettra en l'intégrant de connaître la vitesse du segment et donc sa nouvelle position. Nous itérons ainsi jusqu'à avoir un déplacement moyen nul sur une distance d'un motif.

\subsection{Limites de validité du modèle}

Une limitation de ce modèle apparaît lorsque le segment rencontre un pic isolé de la surface. Cela peut arriver lorsque la force de contact n'agit pas ou est insuffisante pour compenser la force appliquée. Dans ce cas-là, la distance entre le segment et la surface est négative ou nulle et le modèle hydrodynamique n'est donc plus valable.

Pour pallier ce problème, nous avons lorsque c'était nécessaire tronqué une partie de la surface en imposant une distance minimale $h_{\min }$ entre les deux surfaces. Cette troncature est temporaire, au temps d'après on reprend la surface discrète et l'on applique de nouveau cette règle de distance minimale (Figure 3).

\subsection{Quelques résultats}

Nous présentons Figure 4 deux exemples d'échantillons de surface de chemise fournis par Renault et Figure 5 deux géométries de segments.

Par les méthodes décrites dans les sections précédentes, nous pouvons obtenir une vision synthétique des performances des deux surfaces pour ce qui concerne la lubrification en construisant les courbes de Stribeck. 


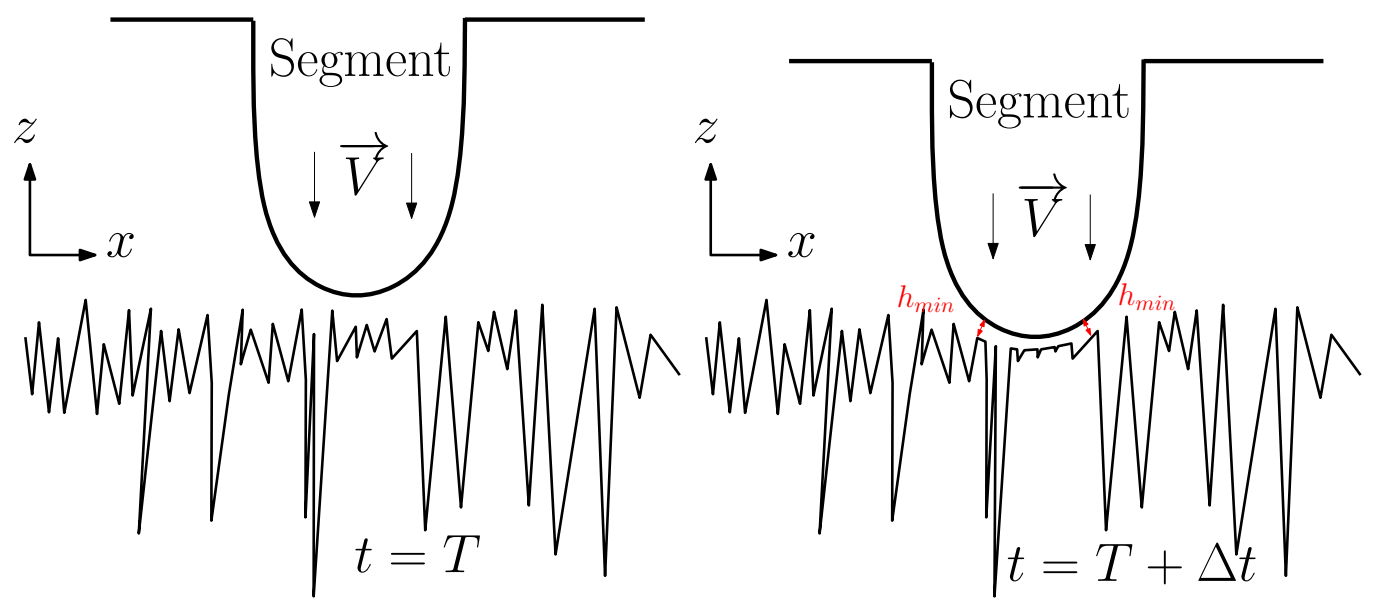

(a) Cas où le modèle est valable

(b) Cas où le modèle JFO ne serait plus valable $h \leq 0$

Figure 3. Exemples où le modèle JFO n'est plus valable

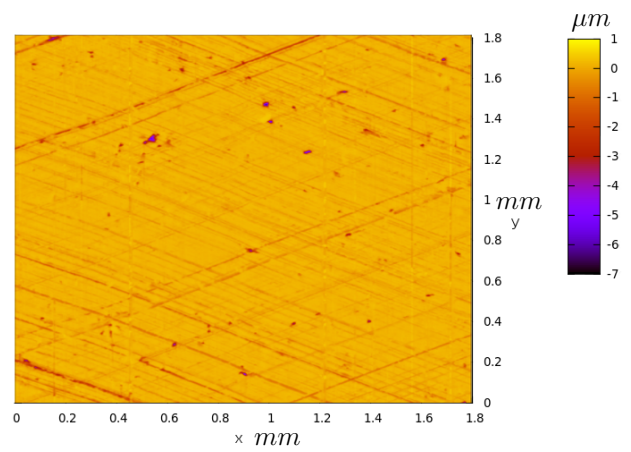

(a) surface L1

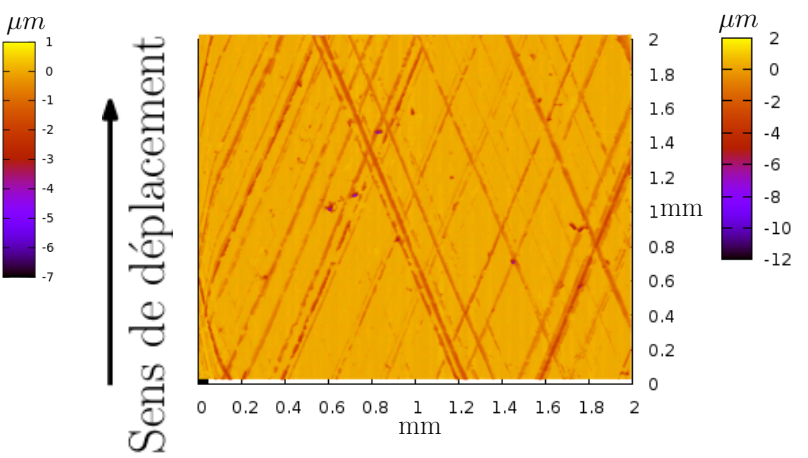

(b) surface L3

Figure 4. Vue des échantillons fournis par Renault

Celles-ci représentent le frottement total en fonction du paramètre $S=\mu u / W_{a}$ où $W_{a}$ est la charge calculée par $W_{a}=\iint_{\Omega} p d x d y$. Ces résultats sont présentés Figures 6 .

\section{Conclusions et perspectives}

Le fait d'avoir voulu prendre des surfaces avec des rugosités réelles était un vrai défi, car d'une part il n'existe pas de méthode fiable et robuste pour dupliquer une surface anisotrope, et d'autre part le modèle de Greenwood Tripp peut ne plus être valable, la surface ne présentant pas forcément une répartition gaussienne des aspérités de plateaux; et dès lors qu'un pic isolé rencontre la surface du segment, le modèle hydrodynamique JFO n'a plus de sens [4]. 


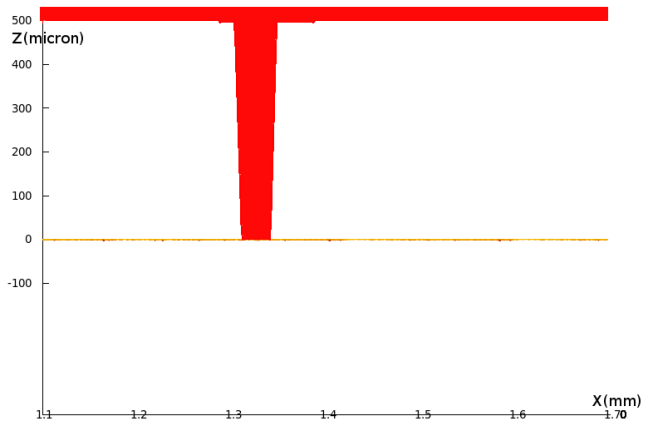

(a) segment plat

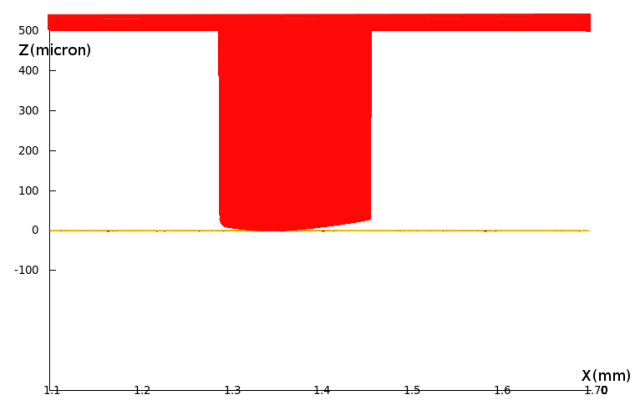

(b) segment coupe-feu

Figure 5. Vue des segments fournis par Renault

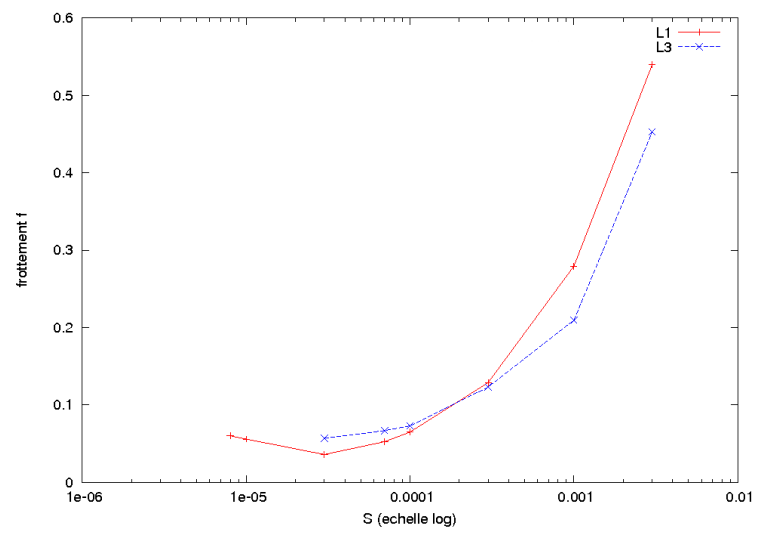

(a) avec un segment plat type racleur

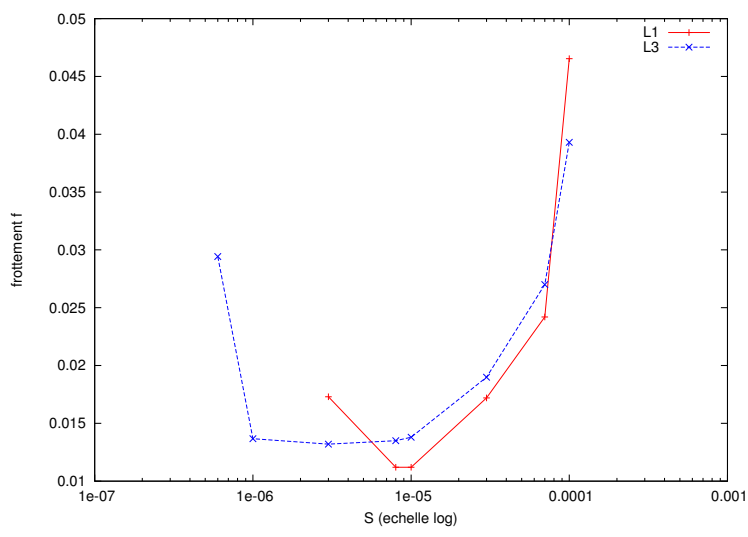

(b) avec un segment coupe feu

Figure 6. Comparaison des surfaces L1 et L3 pour différents segments

Nous avons proposés diverses techniques afin de dupliquer une surface par motif et d'extraire les données nécessaire au modèle de contact de Greenwood Tripp [6].

Il faut aussi se poser la question de la pertinence des variables statistiques du modèle de contact. Comment avoir de manière unique un plan moyen et un écart type des rugosités de plateaux ayant une répartition gaussienne? De plus, ces résultats sont très influencés par la constante $K_{T}$ qui varie énormément dans la littérature de 2.5E7 [4] à 6.49E10 [8], et qui est difficile à évaluer.

D'autre part le modèle JFO "moyenne" le comportement du fluide à travers le "gap" et ne permet pas de déterminer la hauteur effective du lubrifiant. D'autres modèles hydrodynamiques [9] ont été proposés pour pallier cette difficulté. 


\section{RÉFÉRENCES}

[1] D. E. Richardson, 2000 , Review of Power Cylinder Friction for Diesel Engines, in Journal of Engineering for Gas Turbines and Power, volume 122, number 4, pp. 506-51, doi :10.1115/1.1290592, ASME, http ://link.aip.org/link/ ?GTP/122/506/1.

[2] D. Dowson, 1998, History of Tribology, Wiley-Blackwell.

[3] Reynolds, O, 1886, On the theory of the lubrication and its application to Mr Beauchamp Tower's experiments, including an experimental determination of the viscosity of th olive oil , in Phil. Trans R., volume 177.

[4] Greenwood, J. A.; Tripp, J. H., 1971, The contact of two nominally flat rough surfaces, in Proceedings of the Institution of Mechanical Engineers, volume 185, pp. 625-633, doi :10.1243/PIME_PROC_1970_185_069_02.

[5] A. Kumar and J. F. Booker, 1991, A finite Element cavitation algorithm, in Transactions of the ASME, volume 113, pp. 276-286

[6] Dalissier, E., 2012, Optimisation des états de surface et nouveau modèle de cavitation pour un écoulement lubrifié, INSA Lyon

[7] Ausas, R. F.; Jai, M.; Buscaglia, G. C., 2009 , A Mass-Conserving Algorithm for Dynamical Lubrication Problems With Caviation in ASME, volume 131.

[8] Organisciak, M., 2007, Optimisation de la microgeometrie des chemises de moteurs a combustion interne, INSA Lyon

[9] G.Buscaglia, I.Ciuperca, E.Dalissier, M.Jai, "A new cavitation model in lubrication : the case of two-zone cavitation" Journal of Engineering Mathematics, accepté en mars 2012. 